\title{
Interaction between thermal efficiency and dynamic controllability for heat-integrated reactors
}

\author{
Yih-Hung Chen, Cheng-Ching $\mathrm{Yu} *$ \\ Department of Chemical Engineering, National Taiwan University of Science Technology, Taipei 106-07, Taiwan, ROC
}

\begin{abstract}
The objective of this work is to quantify the relationship between thermal efficiency and dynamic controllability. For a given feed-effluent heat exchanger (FEHE), relationships between the degree of heat recovery and open-loop poles are derived. Then, similar to the ultimate gain in the control literature, the ultimate heat recovery can be derived. By ultimate heat recovery, we mean the amount of heat recovery such that the open-loop system is at the limit of stability (i.e. having poles at the imaginary axis). Since the controllability is characterized by the pole locations, achievable closed-loop performance of heat integrated systems can also be evaluated. Internal as well as external energy load disturbances are compared. The results clearly quantify the trade-off between the thermal efficiency and controllability. More importantly, the results presented here offer a simple way to synthesize a controllable heat integrated reactor. (C) 2000 Elsevier Science Ltd. All rights reserved.
\end{abstract}

Keywords: Feed-effluent heat exchanger; Thermal efficiency; Dynamic controllability

\section{Introduction}

Chemical processes generally are thermally inefficient. Based on second law analysis, the efficiency of the majority of chemical processes is in the range of $20-30 \%$ (Seider, Seader \& Lewin, 1999). There exists an incentive for improvement by heat integration. This is accomplished by controlling energy flows between process streams via heat exchangers. Unfortunately, many examples in the literature show that the thermal feedback makes the plants more difficult to control (Douglas, Orcutt \& Berthiaume, 1962; Silverstein \& Shinnar, 1982; Tyreus \& Luyben, 1993; Morud \& Skogestad, 1998). This coincides with one's intuition that reversible processes are difficult to control (e.g. the driving force is smaller and the handle for control is weaker). The objective of this work is to quantify the relationship between thermal efficiency and dynamic controllability.

The process of interest is heat integrated reactors (feed-effluent heat exchanger, FEHE). Luyben, Tyreus and Luyben (1998) probably is the first study on the effect of design on the control of FEHE. This heat

\footnotetext{
* Corresponding author. Fax: + 886-2-27376644.
}

E-mail address: ccyu@ch.ntust.edu.tw (C.-C. Yu) integrated process is nicely analyzed using the heat generation and removal curves. In this work, a more control oriented approach is taken to look at the interaction between design and control. First, relationships between the degree of heat recovery and openloop poles are derived. Then, similar to the ultimate gain in the control literature, the ultimate heat recovery can be derived. By ultimate heat recovery, we mean the amount of heat recovery such that the open-loop system is at the limit of stability (i.e. having poles at the imaginary axis). Since the system dynamics is characterized by the pole locations, achievable closed-loop performance of heat integrated reactors can also be evaluated. Next, disturbance rejection capability of FEHE is explored. Both internal and external heat load changes are compared. The effects of by-pass, addition of boiler and/or furnace are also studied and implication in design is also explored.

\section{Feed-effluent heat exchanger (FEHE)}

Consider an adiabatic plug flow reactor with exothermic reactions. The reactor feed is, first, heated to the reaction level and outlet stream is then cooled to prevent undesirable operation conditions. The repeated 


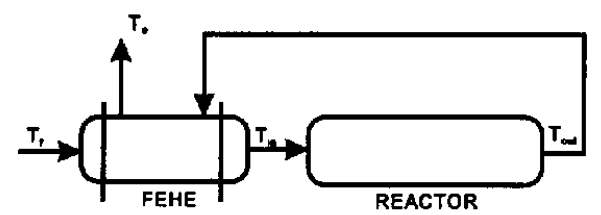

Fig. 1. Reactor with feed-effluent heat exchanger.

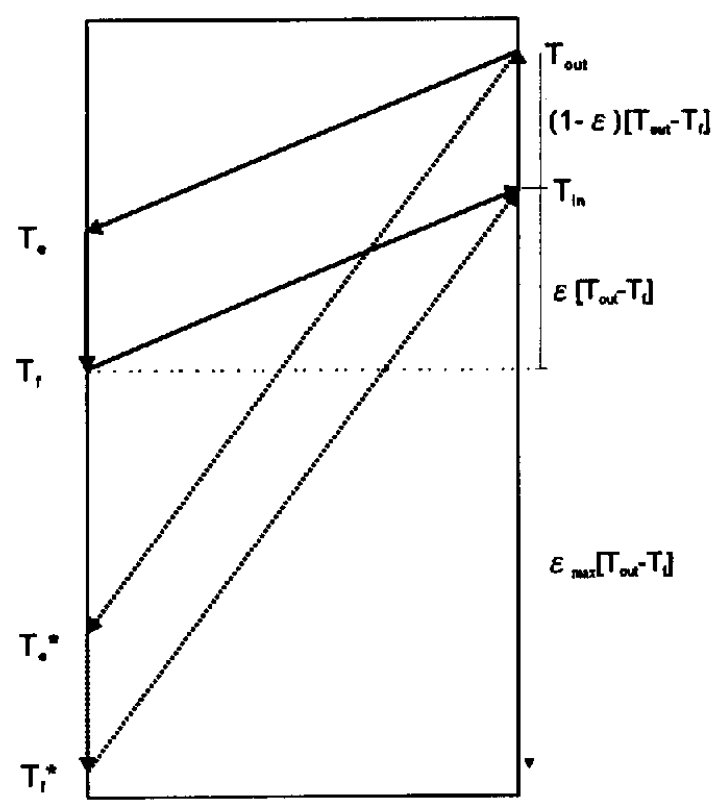

Fig. 2. Temperature profile across heat exchanger with two different effectiveness, $\varepsilon$ and $\varepsilon_{\max }$.

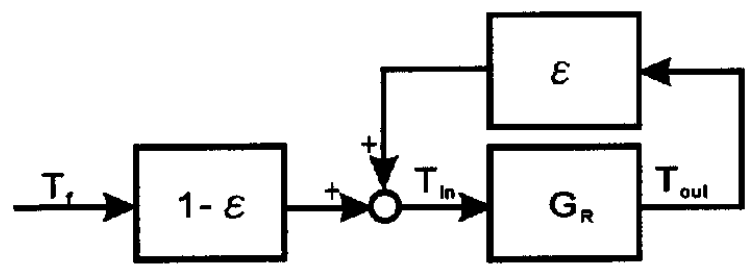

Fig. 3. Block diagram for the heat-integrated reactor of Fig. 1.

heating and cooling makes the plant thermally inefficient. The efficiency problem can be improved significantly by pre-heating the feed to the reaction temperature $\left(T_{\text {in }}\right)$ with the reactor outlet stream $\left(T_{\text {out }}\right)$ as shown in Fig. 1. This is the scheme of a FEHE coupled with an adiabatic reactor, called FEHE system hereafter, which can be seen in many process flowsheets.

\subsection{Thermal efficiency}

Since a heat exchanger is one of the major process unit in the loop, the effectiveness $(\varepsilon)$ is used for the modeling (Luyben et al., 1998). Temperature across a FEHE can be expressed in a graph similar to the temperature profiles along a countercurrent heat ex- changer as shown in Fig. 2. The feed $\left(T_{\mathrm{f}}\right)$ is heated up to the reaction temperature $\left(T_{\text {in }}\right)$ and after the exothermic reaction, the reactor outlet temperature is increased to $T_{\text {out }}$. This reactor outlet stream is utilized to heat up the feed and the temperature of the exit stream is cooled down to $T_{\mathrm{e}}$. Assuming the heat capacities are the same, i.e. $C_{p, H}=C_{p, C}$, after some algebraic manipulation, the ratio of heat recovered to heat generated is simply:

$\frac{Q_{\text {rec }}}{Q_{\text {gen }}}=\frac{\varepsilon}{1-\varepsilon}$

Eq. (1) indicates that the system is more thermally efficient as $\varepsilon$ increases. Notice that the ratio can be greater than one, furthermore, there exists an upper limit for the effectiveness. That is, the absolute temperature of the heat sink is the surrounding temperature $T_{0}$ (Seider et al., 1999).

\subsection{Dynamic controllability}

Linear theory is used to analyze the FEHE. The block diagram of the FEHE (Fig. 3) shows a positive feedback system with a gain of $\varepsilon$ and a disturbance gain of $1-\varepsilon$ (Luyben, 1998). Assume that the dynamics of the heat exchanger is negligible. The reactor inlet temperature $\left(T_{\text {in }}\right)$ is related to the feed temperature and the reactor outlet temperature by the following equation:

$T_{\text {in }}(s)=\varepsilon T_{\text {out }}(s)+(1-\varepsilon) T_{\mathrm{f}}(s)$

The reactor is assumed to be a simple open-loop stable system with a time constant $\tau_{\mathrm{R}}$ and a reactor gain $K_{\mathrm{R}}$ (this assumption will be relaxed in a later section).

$G_{\mathrm{R}}(s)=\frac{T_{\text {out }}}{T_{\text {in }}}=\frac{K_{\mathrm{R}}}{\tau_{\mathrm{R}} s+1}$

Combining Eq. (2) Eq. (3) gives:

$\frac{T_{\text {in }}}{T_{\mathrm{f}}}=\frac{1-\varepsilon}{1-\varepsilon G_{\mathrm{R}}}=\frac{\left(1-\varepsilon / 1-\varepsilon K_{\mathrm{R}}\right)\left(\tau_{\mathrm{R}} s+1\right)}{\left(\tau_{\mathrm{R}} / 1-\varepsilon K_{\mathrm{R}}\right) s+1}$

The coupled open-loop system has a pole at $p=$ $\left(\varepsilon K_{\mathrm{R}}-1\right) / \tau_{\mathrm{R}}$. Several observations can be made immediately.

1. The open-loop pole $p$ moves to the right-half-plane (RHP) as percent of energy recovery increases (i.e. as $\varepsilon$ increases) as shown in Fig. 4.

2. Similar to the ultimate gain in process control, there exists an ultimate effectiveness $\left(\varepsilon_{\mathrm{u}}=1 / K_{\mathrm{R}}\right)$ in the design of FEHE where the system is at the limit of stability.

3. An unstable pole imposes an inherent limitation on achievable performance.

The trade-off between dynamic controllability (pole location) and thermal efficiency $(\varepsilon)$ is nicely illustrated in the FEHE example. As we try to recover more and 


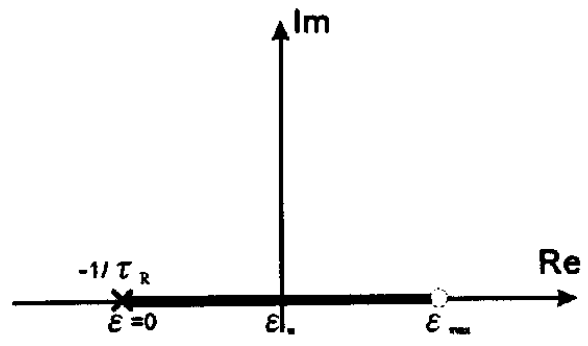

Fig. 4. Open-loop pole of heat-integrated reactor with different degree of heat-integration.

more heat, the open-loop pole simply moves toward the RHP. In other words, we can visualize the effect of process design on dynamic controllability (Fig. 4).

\section{Design and control}

\subsection{Process}

Let us use a linear version of the $\mathrm{C}_{4}$ isomerization process (Luyben et al., 1998) to illustrate the interaction between design and control. This is a mild exothermic reaction and, for the given conversion $(\xi=62 \%)$, the $\mathrm{PFR}$ gives a temperature rise of $42^{\circ} \mathrm{F}$ and a $K_{\mathrm{R}}=1.732$. The control objective is to maintain the reactor inlet temperature $T_{\text {in }}$ by changing the heat input to the furnace $Q_{\mathrm{F}}$. Four cases, corresponding to different degrees of energy recovery, are studied. In all the four cases, the feed temperature and the reactor inlet temperature are kept constant. In case 1,97\% of the energy required to heat up the feed comes from the FEHE (i.e.

Table 1

FEHE with different degrees of heat recovery

\begin{tabular}{lllrl}
\hline Case & $\varepsilon$ & Recovery (\%) & \multicolumn{1}{l}{ Pole } & Remark \\
\hline 1 & 0.68 & 97 & 1.03 & Unstable \\
2 & 0.58 & 83 & 0.01 & Unstable \\
3 & 0.57 & 82 & -0.01 & Stable \\
4 & 0.21 & 30 & -3.73 & Stable \\
\hline
\end{tabular}

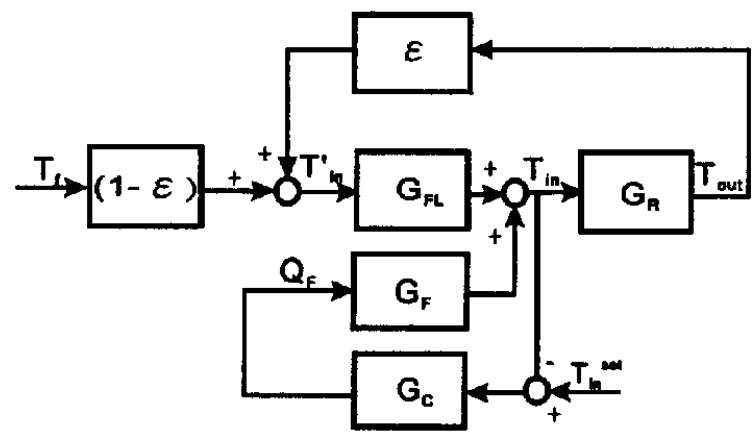

Fig. 5. Block diagram for the heat-integrated reactor with the reactor inlet temperature controlled by furnace heat input.
$3 \%$ of the energy provided by the furnace). The percentage decreases to 83,82 and 30 for cases 2,3 and 4, respectively. From the stability point of view, cases 1 and 2 are open-loop unstable and cases 3 and 4 are stable. Table 1 summarizes the percent of energy recovery and corresponding pole locations.

Since the furnace is used to control $T_{\text {in }}$, the block diagram is a little different from the previous one (Fig. 5). Let us assume the furnace has a first order dynamics.

$G_{\mathrm{F}}(s)=\frac{T_{\text {in }}}{Q_{\mathrm{F}}}=\frac{K_{\mathrm{F}}}{\tau_{\mathrm{F}} s+1}$

The disturbance gain for the furnace $\left(G_{\mathrm{FL}}(0)=K_{\mathrm{FL}}\right)$ is assumed to be one (In reality, it is a little smaller than one because the heat capacity increases with the temperature.). The reactor dynamics is also a first order system (Eq. (3)). Therefore, we can derive for the open-loop transfer functions under positive feedback.

$$
\begin{aligned}
T_{\text {in }}= & \frac{G_{\mathrm{F}}}{1-\varepsilon K_{\mathrm{FL}} G_{\mathrm{R}}} Q_{\mathrm{F}}+\frac{(1-\varepsilon) K_{\mathrm{FL}}}{1-\varepsilon K_{\mathrm{FL}} G_{\mathrm{R}}} T_{\mathrm{f}} \\
= & \frac{\left(K_{\mathrm{F}} / 1-\varepsilon K_{\mathrm{FL}} K_{\mathrm{R}}\right)\left(\tau_{\mathrm{R}} s+1\right)}{\left(\tau_{\mathrm{R}} / 1-\varepsilon K_{\mathrm{FL}} K_{\mathrm{R}}\right)(s+1)\left(\tau_{\mathrm{F}} s+1\right)} Q_{\mathrm{F}} \\
& +\frac{(1-\varepsilon) K_{\mathrm{FL}} /\left(1-\varepsilon K_{\mathrm{FL}} K_{\mathrm{R}}\right)\left(\tau_{\mathrm{R}} s+1\right)}{\tau_{\mathrm{R}} /\left(1-\varepsilon K_{\mathrm{FL}} K_{\mathrm{R}}\right)(s+1)} T_{\mathrm{f}} \\
= & G_{\mathrm{p}}(s) Q_{\mathrm{F}}+G_{\mathrm{L}}(s) T_{\mathrm{f}}
\end{aligned}
$$

Notice that this is a strictly proper system in the process transfer function $G_{\mathrm{p}}$ and a proper system in the load transfer function $G_{\mathrm{L}}$ (e.g. the net order is zero). Again, the design (value of $\varepsilon$ ) determines the stability of the open-loop system. The ultimate effectiveness then becomes $\varepsilon_{\mathrm{u}}=1 / K_{\mathrm{FL}} K_{\mathrm{R}}$ which is effectively the same as the previous case because $K_{\mathrm{FL}} \cong 1$.

\subsection{Control system design}

A systematic procedure is employed to evaluate performance of different designs. The internal model control (IMC) principle is used to derive corresponding PID controllers (i.e. IMC-PID design). For the stable systems (cases 1 and 2), we are dealing with a second order lead/lag systems (net order of one). For the open-loop stable system, a first order IMC filter is assumed.

$F(s)=\frac{1}{\lambda s+1}$

Here $\lambda$ is the filter time constant, the controller $K(s)$ is shown in Table 2. For the unstable system, again, the IMC principle is employed (Rotstein \& Lewin, 1991). Following Rotstein and Lewin, a second order IMC filter is used.

$F(s)=\frac{r s+1}{(\lambda s+1)^{2}}$ 
Table 2

PID controller parameters for stable and unstable cases

\begin{tabular}{lllll}
\hline & $K \mathrm{c}^{\mathrm{a}}$ & $\tau_{\mathrm{I}}$ & $\tau_{\mathrm{D}}$ & $\mathrm{Lag}$ \\
\hline Stable & $\left(\tau_{\mathrm{R}}^{\prime}+\tau_{\mathrm{F}}\right) /\left(K_{\mathrm{p}} \lambda\right)$ & $\left(\tau_{\mathrm{R}}^{\prime}+\tau_{\mathrm{F}}\right)$ & $\tau_{\mathrm{R}}^{\prime} \tau_{\mathrm{F}} /\left(\tau_{\mathrm{R}}^{\prime}+\tau_{\mathrm{F}}\right)$ & $\tau_{\mathrm{R}}$ \\
Unstable & $\tau_{\mathrm{R}}^{\prime}\left(r+\tau_{\mathrm{F}}\right) /\left(K_{\mathrm{p}} \lambda^{2}\right)$ & $\left(r+\tau_{\mathrm{F}}\right)$ & $r \tau_{\mathrm{F}} /\left(r+\tau_{\mathrm{F}}\right)$ & $\tau_{\mathrm{R}}$ \\
\hline
\end{tabular}

" $K \mathrm{p}= \pm\left(K_{\mathrm{F}}\right) /\left(\mathrm{I}-\varepsilon K_{\mathrm{FL}} K_{\mathrm{R}}\right)$ and $\tau_{\mathrm{R}}^{\prime}= \pm\left(\tau_{\mathrm{R}}\right) /\left(\mathrm{I}-\varepsilon K_{\mathrm{FL}} K_{\mathrm{R}}\right)$ with ' + ' for stable and ' - ' for unstable systems, $r=\lambda\left(\lambda / t_{\mathrm{R}}^{\prime}+2\right)$.

Table 3

Closed-loop process and load transfer functions for the FEHE

\begin{tabular}{|c|c|c|}
\hline & Stable & Unstable \\
\hline$G_{p} G_{c}\left(1+G_{p} G_{c}\right.$ & $\frac{1}{\left(\frac{\tau_{\mathrm{R}}}{x\left(1-\varepsilon K_{\mathrm{FL}} \mathcal{K}_{\mathrm{R}}\right)}\right) s+1}$ & 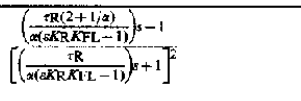 \\
\hline \multirow[t]{2}{*}{$G_{\mathrm{L}} / 1+G_{\mathrm{p}} G_{\mathrm{s}}$} & $\frac{\tau_{\mathrm{R}}}{\alpha\left(1-i K_{\mathrm{R}} K_{\mathrm{FL}}\right)} \frac{(1-\varepsilon) K_{\mathrm{FL}}}{1-\varepsilon K_{\mathrm{K}} K_{\mathrm{FL}}} s\left(\tau_{\mathrm{R}} s+1\right)$ & $=(1-\varepsilon) K_{F L} s\left(\tau_{R} s+1\right)$ \\
\hline & {$\left[\left(\frac{\tau_{\mathrm{R}}}{\alpha\left(1-\varepsilon K_{\mathrm{R}} K_{\mathrm{F}}\right)}\right) s+1\right]\left[\left(\frac{\tau_{\mathrm{R}}}{\left(1-\varepsilon K_{\mathrm{R}} K_{\mathrm{FL}}\right.}\right) s+1\right]$} & $\frac{\alpha\left(\varepsilon K_{\mathrm{R}} K_{\mathrm{FL}}-1\right)_{\varepsilon_{\mathrm{R}} K_{\mathrm{FL}}-1}}{\left[\left(\frac{\mathfrak{i}_{\mathrm{R}}}{\alpha\left(\Sigma K_{\mathrm{R}} K_{\mathrm{FL}}-1\right)}\right) s+1\right]^{2}}$ \\
\hline
\end{tabular}
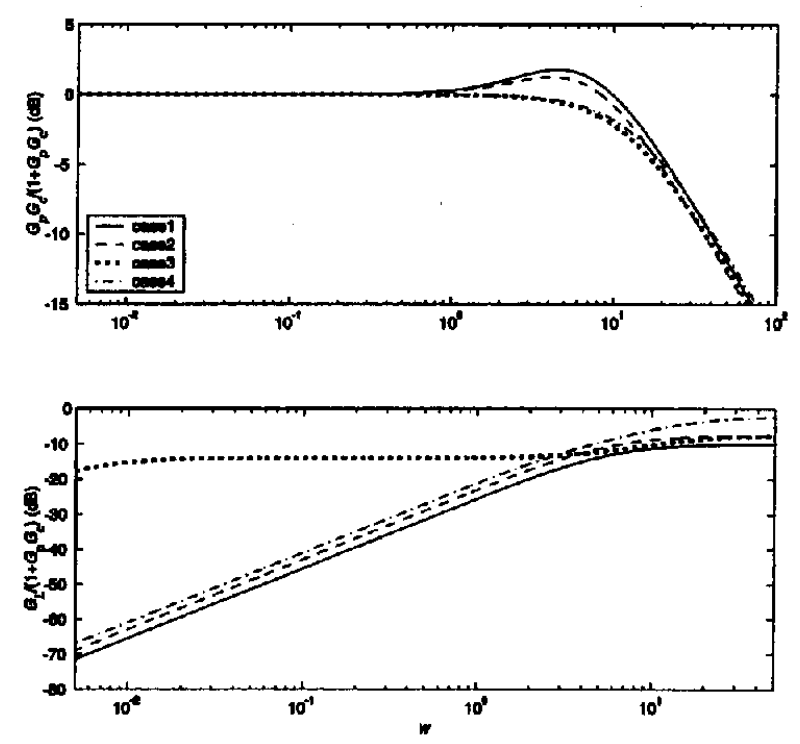

Fig. 6. Bode plots of the complementary sensitivity function, sensitivity function and closed-loop load transfer function (TLC) for four different cases in Table 1.

and $r$ relates to $\lambda$ and $G_{\mathrm{p}}$ in the following way, $r=\lambda(\lambda)$ $\left.\tau_{\mathrm{R}}^{\prime}+2\right)$. Table 2 gives the PID controller parameters.

If the filter time constant is set to a ratio of dominant time constant $\tau_{\mathbf{R}}^{\prime}$, i.e. $\lambda=\tau_{\mathbf{R}}^{\prime} / \alpha$, the performance mea- sures can be expressed explicitly in terms of the ratio $\alpha$ and process parameters. Table 3 gives the complementary sensitivity function and closed-loop load transfer function for the FEHE when process is stable or unstable.

In this work, $\alpha$ is set to $\left(1-\varepsilon K_{\mathrm{FL}} K_{\mathrm{R}}\right)$ such that the dominant closed-loop time constant is equal to the reactor time constant (i.e. $\lambda=\tau_{\mathrm{R}}$ ). This provides a common basis to compare different designs.

\subsection{Analysis}

In a heat integrated FEHE, two type of disturbances are identified. The internal disturbance occurs when the source of the disturbance is in the positive feedback loop. The reactor (inlet) temperature set point change is a typical example. Another example is the change in the heat of reaction. The external disturbance, on the other hand, means the source of disturbance comes from outside. For example, temperature variations in the feed stream $\left(T_{\Gamma}\right)$.

\subsubsection{Internal disturbance}

Since the disturbance occurs at any possible location in the positive feedback loop (Fig. 5), the complementary sensitivity function $(H(s)=G K /(1+G K))$ or the sensitivity function $(S(s)=1 /(1+G K))$ is a good performance measure. As mentioned earlier (e.g. Fig. 4), we expect the closed-loop performance will deteriorate as the percent of energy recovery increases (since, we will be moving the system pole toward the RHP). This is exactly what we obtained in the Bode plots of $H(i \omega)$ as shown in Fig. 6. For the two unstable cases ( 1 and 2), there exist resonant peaks in the closed-loop Bode plots. On the other hand, non-overshoot set point responses are expected for two stable cases ( 3 and 4). Qualitatively, the shapes of the Bode plots remain the same if we tighten the speed of responses (i.e. reducing the filter time constant $\lambda$ ).

Time domain simulations are used to explore the tradeoffs. Again, let us use the linear example with $K_{\mathrm{R}}=1.73$ and $\tau_{\mathrm{R}}=0.17 \mathrm{~min}$. For a step temperature set point change, cases 1 and 2 show an overshoot in $T_{\text {in }}$ while the other two cases ( 3 and 4 ) give rather smooth approach to the new set point (Fig. 7A). Similar results are observed for a step temperature increase in $T_{\text {in }}$ (Fig. 7B).

Frequency domain and time domain analyses reassure one's physical intuition, increased heat integration simply deteriorating control performance. This is, at least, the case when internal disturbance is considered.

\subsubsection{External disturbance}

This kind of load disturbance probably is more important than the previous one. A typical scenario is as follows. The temperature of the feed stream changes as 
a result of upsets from upstream units. This, subsequently, disturbs the reactor inlet temperature $T_{\text {in }}$. Intuitively, we are expecting a similar outcome as the previous case, performance degradation as a result of heat integration. A further analysis shows otherwise.

The closed-loop load transfer function $\left(G_{\mathrm{L}, \mathrm{CL}}(s)=\right.$ $\left.G_{\mathrm{L}} /(1+G K)\right)$ in Table 3 indicates this is a proper system. Therefore, we can find the high frequency asymptote for $G_{\mathrm{L}, \mathrm{CL}}$.
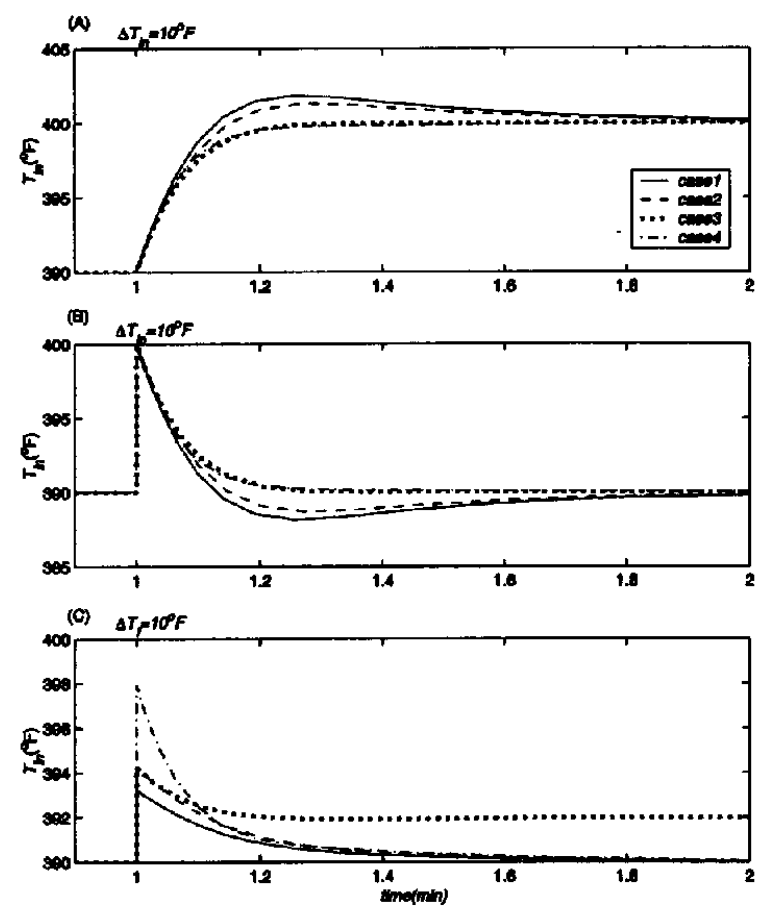

Fig. 7. Control performance for four different cases under, (A) step set point change in $T_{\text {in }}$; (B) step increase in $T_{\text {in }}$; and (C) step change in inlet temperature $\left(T_{f}\right)$.

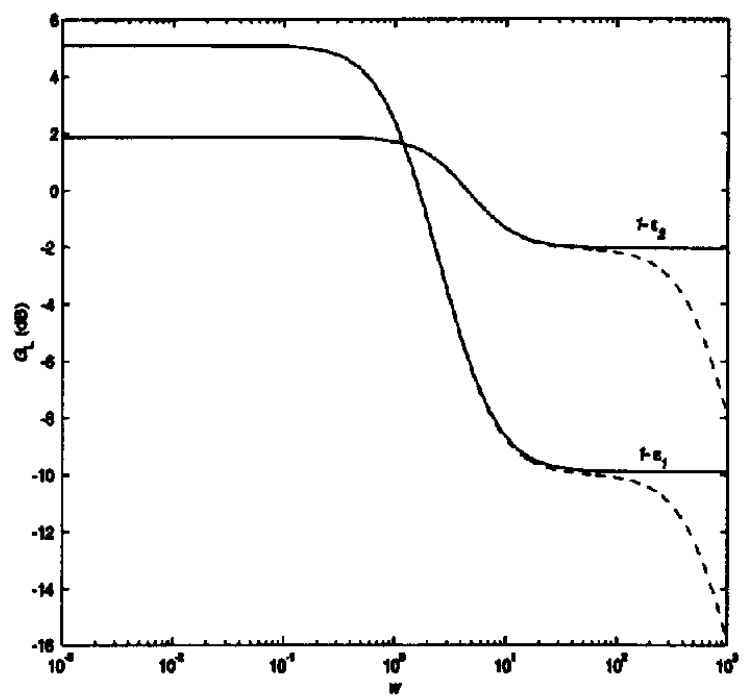

Fig. 8. Bode plot and corresponding high frequency behavior of GL with negligible heat exchanger dynamics (solid) and first order dynamics (dashed).
$G_{\mathrm{L}, \mathrm{CL}}(s)=\lim _{s \rightarrow \infty}\left[G_{\mathrm{L}, \mathrm{CL}}(s)\right]=(1-\varepsilon) K_{\mathrm{FL}}$

Since $K_{\mathrm{FL}}$ is close to unity, the high frequency asymptote is effectively takes the value of $(1-\varepsilon)$. Note that an easier way to find the asymptote is simply taking the limit of the open-loop transfer function $\left(G_{\mathrm{L}}\right.$ in Eq. (6)), because the high frequency asymptote of $1 /(1+G K)$ is unity. Several observations can be made immediately. First, the value of the asymptote becomes smaller as the percent of energy recovery becomes larger. Second, it is a system inherent property. In other words, it remains the same regardless of how the controller is designed (as long as the integral action is used).

Notice that the high frequency asymptote, in essence, is similar to the tyreus load-rejection capability (TLC) (Luyben \& Luyben, 1997) which indicates the ultimate load rejection capability. Fig. 6 indicates that as we try to recover more and more heat (larger $\varepsilon$ ), the high frequency asymptote $(1-\varepsilon)$ becomes smaller. This implies improved external load rejection can be achieved for heat integrated systems and better performance is expected if one tries to recover more heat. Since only high frequency information is used, the performance generally corresponds to the initial response in time domain. Fig. 7C clearly indicates that case $1(\delta=0.68)$ possesses the best disturbance rejection capability for a step change in the feed temperature and case D $(\varepsilon=$ $0.21)$ shows the highest peak temperature for the same disturbance. Furthermore, it is interesting to note that cases 2 and 3, showing almost the same responses for internal load change, give completely different responses (Fig. 7). The temperature in case 3 takes extremely long time $(800 \mathrm{~min})$ to return to the set point. This is the case where the open-loop pole is very close to the imaginary axis but still in the LHP. Since the load transfer function $\left(G_{\mathrm{L}}\right.$ in Eq. (6)) shares the same pole, a step change in $T_{\text {in }}$ acts as a ramp type (type 2) of disturbance and consequently leads to a very sluggish response.

As opposed to one's intuition, on-going analyses indicate that heat integrated system exhibits better disturbance rejection capability for external load changes. The reason is, heat integrated system gives better high frequency behavior for closed-loop load transfer function. This example illustrates the importance to look at some kind of load rejection measure such as TLC (it cannot be seen for sensitivity or complementary sensitivity function).

Up to this point, we assume the dynamics of the heat exchanger is negligible. If the FEHE shows a first order dynamics with a time constant of $\tau_{\mathrm{H}}$, then the openloop load transfer function (for the coupled system) is no longer a proper system. Assuming that $\tau_{\mathrm{H}}$ is $1 / 10$ of $\tau_{\mathrm{R}}$ and $K_{\mathrm{FL}}$ is unity, the load transfer function then becomes (Fig. 8): 
$G_{\mathrm{L}}(s)=\frac{(1-\varepsilon)\left(\tau_{\mathrm{R}} s+1\right)}{\left(\tau_{\mathrm{H}} s+1\right)\left(\tau_{\mathrm{R}} s+1\right)-\varepsilon K_{\mathrm{R}}}$

The high frequency asymptotes no longer exist as shown in the dashed lines in Fig. 8. However, $(1-\varepsilon)$ corresponds to the high frequency plateau between the frequency range of $1 / \tau_{\mathrm{R}}$ and $1 / \tau_{\mathrm{H}}$ as shown in Fig. 8 . Again, it also determines the ultimate load rejection performance, since this is the frequency range we really are interested in.

\section{Conclusion}

In this work, heat integrated reactors are used to illustrate the effect of heat integration on dynamic controllability. Root locus plots of the effectiveness (as a measure of thermodynamic efficiency) can be used to visualize the effect of design on control. The concept of ultimate effectiveness $\left(\varepsilon_{\mathrm{u}}\right)$ is proposed which served as an indicator of open-loop stability limit for given process design. A systematic control system design procedure is also proposed to evaluate closed-loop performance. Results are as follows:

1. as expected, increased heat integration deteriorates control performance under internal disturbances (e.g. set point changes, unexpected heat generation or removal within the positive feedback loop etc.) The reason for that is: heat integration moves the open-loop pole toward the RHP; and

2 . as opposed to one's intuition, increased heat integration shows better performance for external disturbances (e.g. amount of energy coming into the system and changes in the feed temperature is a typical example). Quantitative explanation for this is, the high frequency behavior (or asymptote) of the load transfer function is improved as we increase heat integration. It can also be understood qualitatively, since external energy input only accounts for fraction of the energy required for the system.

\section{Acknowledgements}

This work is supported by the National Science Council of Taiwan.

\section{References}

Douglas, J. M., Orcutt, J. C., \& Berthiaume, P. W. (1962). Design and control of feed-effluent exchanger-reactor systems. Industrial Engineering \& Chemical Fundamentals, 1, 253.

Luyben, W. L. (1998). External versus internal open-loop unstable processes. Industrial Engineering \& Chemical Research, 37, 2713.

Luyben, W. L., \& Luyben, M. L. (1997). Essentials of process control. New York: McGraw-Hill.

Luyben, W. L., Tyreus, B. D., \& Luyben, W. L. (1998). Plantwide process control. New York: McGraw-Hill.

Morud, J. C., \& Skogestad, S. (1998). Analysis of instability in an industrial ammonia reactor. American Institute of Chemical Engineering Journal, 44, 888.

Rotstein, G. E., \& Lewin, D. R. (1991). Simple PI and PID tuning for open-loop unstable systems. Industrial Engineering \& Chemical Research, 30, 1864.

Seider, W. D., Seader, J. D., \& Lewin, D. R. (1999). Process design principle: synthesis, analysis, and evaluation. New York: Wiley.

Silverstein, J. L., \& Shinnar, R. (1982). Effect of design on the stability and control of fixed bed catalytic reactors with heat feedback. 1. Concept. Industrial Engineering, Chemical Processes Design \& Development, 21, 241.

Tyreus, B. D., \& Luyben, W. L. (1993). Unusual dynamics of a reactor/preheater process with deadtime, inverse response and openloop instability. Journal of Process Control, 3, 241. 